\title{
Comparison of tumor markers using different detection devices
}

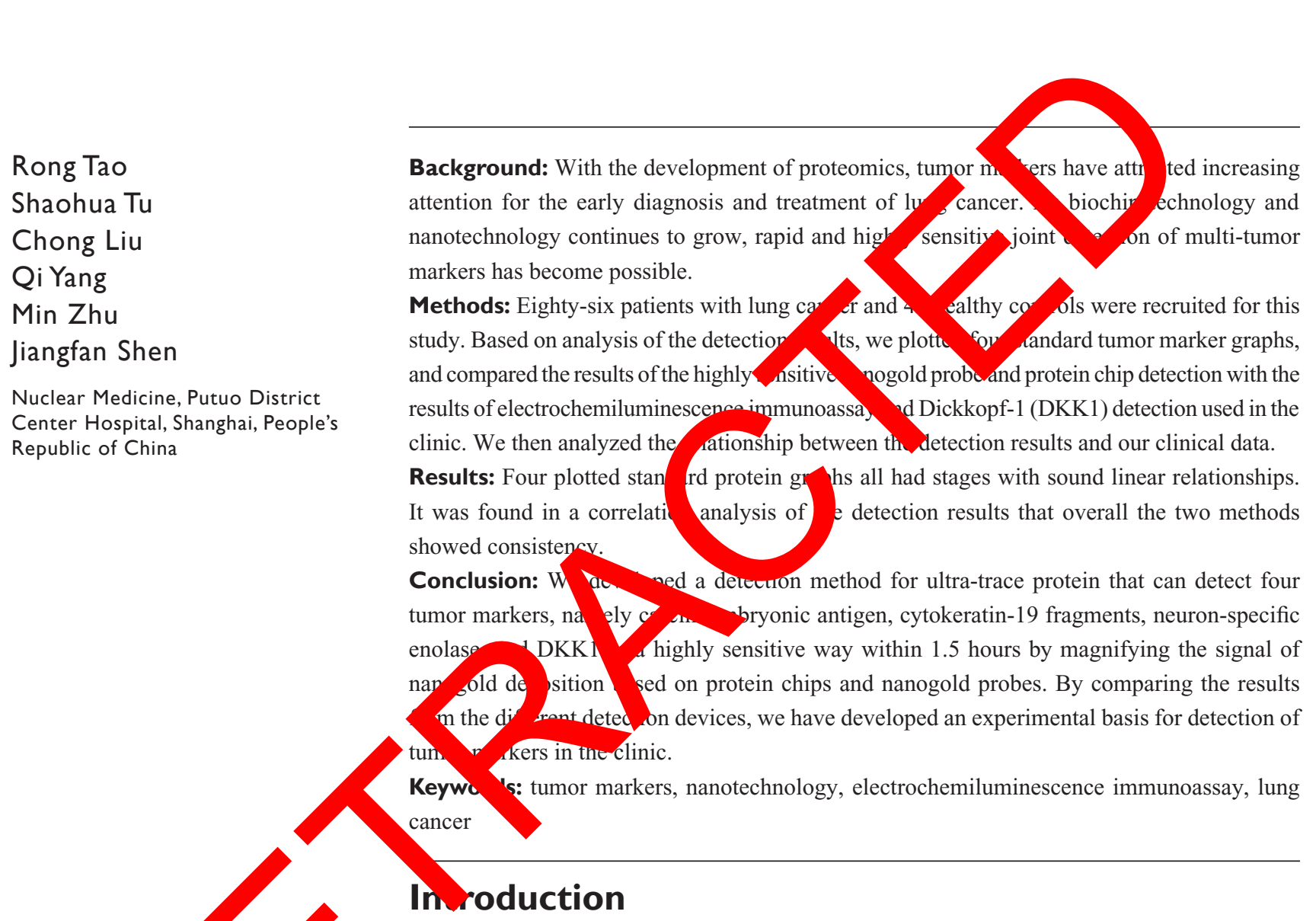

Lung cancer ranks first in the incidence of malignant tumors worldwide. In the People's Republic of China, the morbidity and mortality has leapt to the first in all types of common tumors. ${ }^{1}$ About 600,000 people die of lung cancer every year. Further, the 5-year survival rate of patients with advanced lung cancer is very low. ${ }^{2}$ With the development of medical technology, the ability to diagnose and treat the disease has improved, but is still far short of the needs in clinical practice. It is thought that the most effective way to reduce the mortality of lung cancer is to diagnose and treat the disease as early as possible. In this way, the 5-year survival rate of patients with lung cancer could be over $70 \%$. However, no distinctive clinical symptoms are found in the early stages of lung cancer due to the insidious nature of the disease. Most lung cancers are found in the intermediate or advanced stages, when they are more likely to proliferate and metastasize. The prognosis of lung cancer is closely related to the clinical stage at which treatment is started. Therefore, it is important to identify the insidious symptoms of lung cancer, make an early diagnosis, and start treatment immediately. Currently, the iconography detection method, ie, low-dose spiral computed tomography (CT), is widely used in clinical practice to detect early-stage lung cancer. Low-dose spiral
Correspondence: Jiangfan Shen Nuclear Medicine, Putuo District Center Hospital, Shanghai 200062, People's Republic of China

Email sjf@ibhsedu.com 
CT has high sensitivity when scanning for lung cancer nodules. However, it has the shortcomings of a high rate of false-positive results and being very expensive to perform. A long-term follow-up study in volunteers at high risk of lung cancer in the USA showed a false-positive rate of $21 \%$ for low-dose spiral CT. ${ }^{2}$ Therefore, identifying whether lung cancer nodules are benign or malignant when investigating for early stage-lung cancer is problematic. In the meantime, the diagnosis of lung cancer relies mainly on histopathologic and cytologic examination, which is invasive, impractical for mass screening, and has poor diagnostic accuracy in the early stages of lung cancer. With the development of proteomics, tumor markers have attracted increasing attention for the early diagnosis of the disease.

Tumor markers may be synthesized via gene expression in tumor cells or produced by the response of the organism to a tumor. The appearance and quantitative changes in these markers reflect in occurrence, development, and proliferation of a tumor. These markers include proteins, enzymes, hormones, the product of cancer gene, polyamine. Many studies have shown that tumor markers are of significance in the early-stage detection and diagnosis, clinical staging, pathological typing, response evaluation, monitoring, and prognostication. ${ }^{3}$ Further, there are some data showing th tumor markers could be considered as a predictor of th outcome of targeted therapy for lung cancer advanced stages. ${ }^{4}$ Markers for benign tumo should have high sensitivity and specificity and be s, ple to Currently, there are still no markers for ing can with high sensitivity and specificity. ${ }^{5}$ In clinio ractice, we to use joint detection of multiple tuma mark so as to inrprove the positivity rate for dete on of lung ca er. This joint detection requires sciep analy is and strict screening of appropriate tumor mar so to avoid wasting of medical resources. ${ }^{6}$ How the $\mathrm{t}$ detect nof multiple indicators is quite omplic ed. Th rent detection methods are still in. qquate sensitivity, but with the development rochip technology and nanotechnology, joint detection of ltiple tumor markers with high sensitivity can be achieved.

After the screening of several tumor markers, we selected carcinoembryonic antigen (CEA), cytokeratin-19 fragments (CYFRA21-1), neuron-specific enolase (NSE), and Dickkopf-1 (DKK1) for joint detection. Based on nanogold probe and protein chip technology, the detection antibody was coated on the surface of nanoparticles, and the antigen and antibody were then specifically on the surface of protein chips. After strengthening the signal by deposition of nanogold, a highly sensitive and feasible detection system was developed. The results obtained with this system were then compared with those obtained from the electrochemiluminescence immunoassay (ECLIA) and DDK1 kits presently used in clinical practice. ${ }^{7}$ This aim of this work was to provide an experimental basis for future clinical detection of tumor markers.

\section{Materials and methods Main reagents and devices}

The main reagents used were 2-morpl sulfonic acid, Tween-20, PEG8000, poly ylpyrrolid e, bovine serum albumin (Sigma-Aldrich, St uis, MO, U A), chlorauric acid (Acros Organic Geel, Be. Ym), $C$ A antigen and antibody (Abcam, $\Omega$ mbridg $U K), 0$ R21-1 antigen and antibody (Yem. Mr ow, Rusia), NSE antigen and antibody (N dix Bioc nica, aniainen, Finland), DKK1 antige antibody ( 1 systems, Minneapolis, MN, USA), quality ontrol antibody immunoglobulin G (Ab arr, alldehyde sut rate (Shanghai Baio Technology Co id, Shanghai People's Republic of China), nanogold solt on (15 nm, Shanghai BioServe Co Ltd, Shanghai, Peop Republ of China), nonfat milk powder (Shanghai angon Broruglcal Engineering Technology and Service Co L† hai, People's Republic of China), sucrose (Shangai Lingfeng Chemical Reagents Co Ltd, Shanghai, People's Republic of China), and trihydroxymethyl aminomethane Beijing Dingguo Chansheng Biotech Co Ltd, Bainjing, People's Republic of China).

The main devices used were an ultra-low temperature freezer (MDF-U4086S, Sanyo, Osaka, Japan), an ultravioletvisible spectrophotometer (V670, JASCO, Tokyo, Japan), a fluorescence inverse microscope (BX51, Olympus, Tokyo, Japan), a transmission electron microscope (JEM2100, Olympus), a refrigerated centrifuge (5804R, Eppendorf, Hamburg, Germany), nitrocellulose films with an aperture of $0.22 \mu \mathrm{m}$ (Corning, New York, NY, USA), a chip sampling instrument (ProSys5510A, Cartesian Technologies, Irvine, CA, USA), a simple water purification system (Millipore, Molsheim, France), a hybridization oven (FYY-3, Xinghua Analytical Instrument Factory, Jiangsu Province, People's Republic of China), and an ordinary freezer (Haier Electronics Co Ltd, Qingdao, People's Republic of China).

\section{Serum samples and clinical data}

Serum samples were taken from 86 patients with biopsy-proven lung cancer and 42 healthy controls at Putuo District Center Hospital. All patients signed their informed 
consent before entering the study. The study protocol was approved by the medical ethics committee at our institution. The 86 patients with lung cancer comprised 58 males and 28 females of mean age 61 (range 42-82) years, and the 42 healthy controls comprised 25 males and 17 females of mean age 54 (range $31-68$ ) years. All the patients have not been treated by radiotherapy, chemotherapy and immunotherapy before.

\section{Determination of optimal quantity of protein and preparation of nanogold probes}

Take $500 \mu \mathrm{L}$ of nanogold solution and regulate the $\mathrm{pH}$ of the solution by $\mathrm{K}_{2} \mathrm{CO}_{3}(0.2 \mathrm{~mol} / \mathrm{L}$, to $\mathrm{pH} 8.5-9.0)$, and then divide the solution into five tubes equally. Add the CEA detection antibody of $0.4,0.6,0.8,1.0$, and $1.2 \mu \mathrm{L}$ into the tube, respectively. Keep it stable for 10 minutes at room temperature and then add $10 \mu \mathrm{L}$ of $1 \mathrm{~mol} / \mathrm{L} \mathrm{NaCl}$ into each tube immediately and observe the color of the solution. Take the least protein addition of the solution which remained red, that was, the optimal stable quantity of protein in the $100 \mu \mathrm{L}$ of nanogold solution (the minimum concentration of protein). Repeat the experiment four times to confirm the reliability of experimental results. In a similar way, the optima quantity of detection antibody CYFRA21-1, NSE, and D K1 in the $100 \mu \mathrm{L}$ nanogold solution was availa

Take $2,000 \mu \mathrm{L}$ of nanogold solution the supernatant until the remaining after centrifugation; and then by $\mathrm{K}_{2} \mathrm{CO}_{3}(0.2 \mathrm{~mol} / \mathrm{L})$ to a $\mathrm{p}$ $\mathrm{pH}^{\mathrm{H}} \mathrm{Q} .5-\mathrm{c}-\mathrm{c}$ ate th

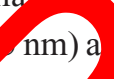
1 remo vition tion into four tubes equally, and th add a certain amount of detection antibody the four tumo narkers; shake up and then place then ato the ybridization oven at $25^{\circ} \mathrm{C}$ for around 1.5 hours; a 11 of $10 \%$ PEG8000, respectively, and then lear alono ernight $4{ }^{\circ} \mathrm{C}$. Twelve hours later, centrifug hese lutions and remove the supernatant again, ply th heavy suspension to set the volume as $50 \mu \mathrm{L}$ finally add $1.5 \mu \mathrm{L} 5 \mathrm{M} \mathrm{NaCl}$, respectively, and keep tho at $4{ }^{\circ} \mathrm{C}$. Transmission electron microscopy was used to obsorve the size and shape of nanogold probes. An ultraviolet-visible spectrophotometer could be applied for scanning analysis and prediction of the concentration of nanogold probes. The centrifugations above were carried at $4^{\circ} \mathrm{C}$ and at 9,000 rpm for 50 minutes.

\section{Fabrication of protein chips}

Firstly, lay the substrate of aldehyde group at the chip place of the chip sampling instrument, then configure the respective antibodies of CEA, CYFRA21-1, NSE, and DKK1, and configure the quality control antibody immunoglobulin $\mathrm{G}$ and spotting solution at the ratio of $1: 1(\mathrm{v} / \mathrm{v})$ on the sample configuration board; next, lay them at the sample board area of chip sampling instrument; turn on the instrument, set the relative programs, and apply spotting according to operational norms. After that, place the protein chips in the incubator and leave for 16 hours at $25^{\circ} \mathrm{C}$ and finally keep it dry at $4^{\circ} \mathrm{C}$.

\section{Immunodetection}

Firstly, keep the solution of 5\% n on protein chips for 10 minutes end the so on of $5 \%$ nonfat milk powder with nanogolo robes and ferent serum samples together and $t^{1}$ add the into th detection area respectively; next, 1 ane soly n into ybridization oven and keep them incus od 45 minrtes; wash chips one or two times in crons; tha dd sor nanogold deposition for dying for vinutes (dy can be carried at $37^{\circ} \mathrm{C}$ or at room temperatur After roughly observing the chip detecti conts by eye, ro the dye solution rapidly and then dd some ultrapure water to stop dying. Observe the result nd signal, re eat dyeing if the signal is too weak.

\section{Anaryurs of results}

1. Sconsidered as positive when there were dark brown points in chips. Usually, the color changes with the diameter of nanogold probes. It tends to be deep when the diameter is large. We could observe the result with the naked eye or by microscopy. Image-Pro Plus 7.0 software was used to analyze the results by calculating optical density. In this way, the indirect quantitative detection of proteins was achieved.

\section{Statistical analysis}

Statistical Package for the Social Sciences version 19.0 software (SPSS Inc, Chicago, IL, USA) was used for the statistical analysis. The data are expressed as the median (interquartile range). The chi-squared test was used to compare rates. The non-parametric test was used to test for differences between the groups. These differences were compared using the Wilcoxon rank sum test, and the difference among three or over three groups was by the Kruskal-Wallis test. $P<0.05$ was considered to be statistically significant.

\section{Results}

\section{Representation of nanogold probes}

Observing the nanogold labeling antibody by transmission electron microscopy, we found that the nanoparticles were of uniform size with an average diameter of $15 \mathrm{~nm}$. 


\section{A}

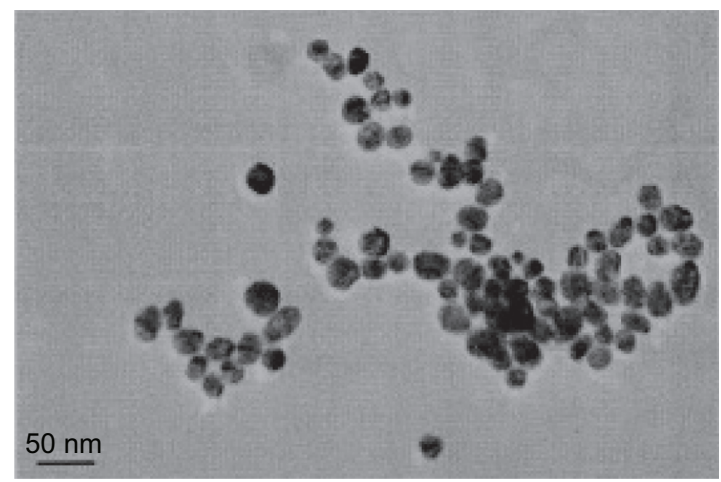

B

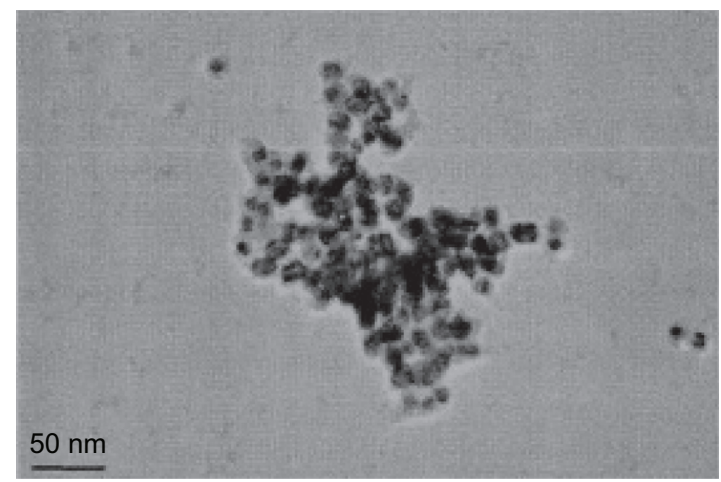

Figure I (A) Unmarked and (B) marked nanogold particles as seen on transmission electron microscopy.

The surrounding interface was quite clear before nanogold marked the antibody (Figure 1A), but after marking the antibody, there were cycles of gray dark aureole in the surrounding interface (Figure 1B), confirming that the nanogold surface had marked the antibody and that the nanoparticles had not become aggregated.

\section{Analysis of specificity of the four tumor markers}

We determined the specificity of the four tumor markers on protein chips separately, and the results are shown Figure 2. The tumor markers showed no cross reaction interruption on the protein chips and had a good specificity. We repeated the experiment four times to en ce th $_{\text {reli- }}$ ability of our results.

\section{Plotting of standard graph}

A standard curve was drawn bas on optical do ity of the detection results, so as to be able to ect the proteins indirectly. The standard ves obtained afto letecting the

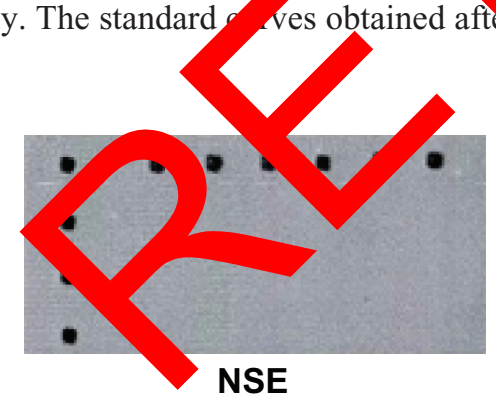

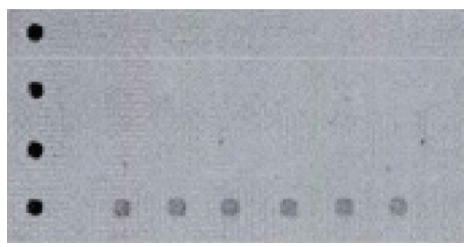

DKK1
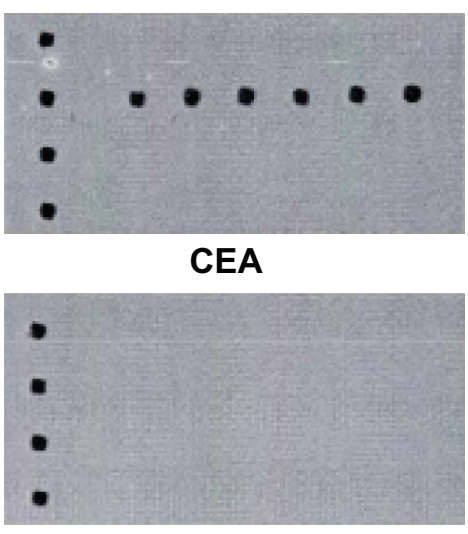

Blank control

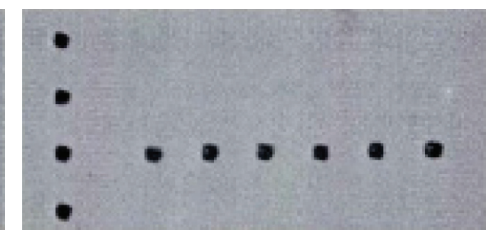

CYFRA21-1

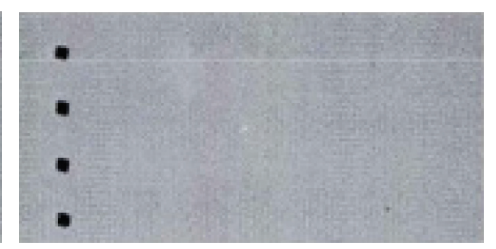

Healthy donors control

Figure 2 Results of analysis of these four specific tumor markers.

Abbreviations: CEA, carcinoembryonic antigen; CYFRA2I-I, cytokeratin-19 fragments; NSE, neuron-specific enolase; DKKI, Dickkopf-I. 

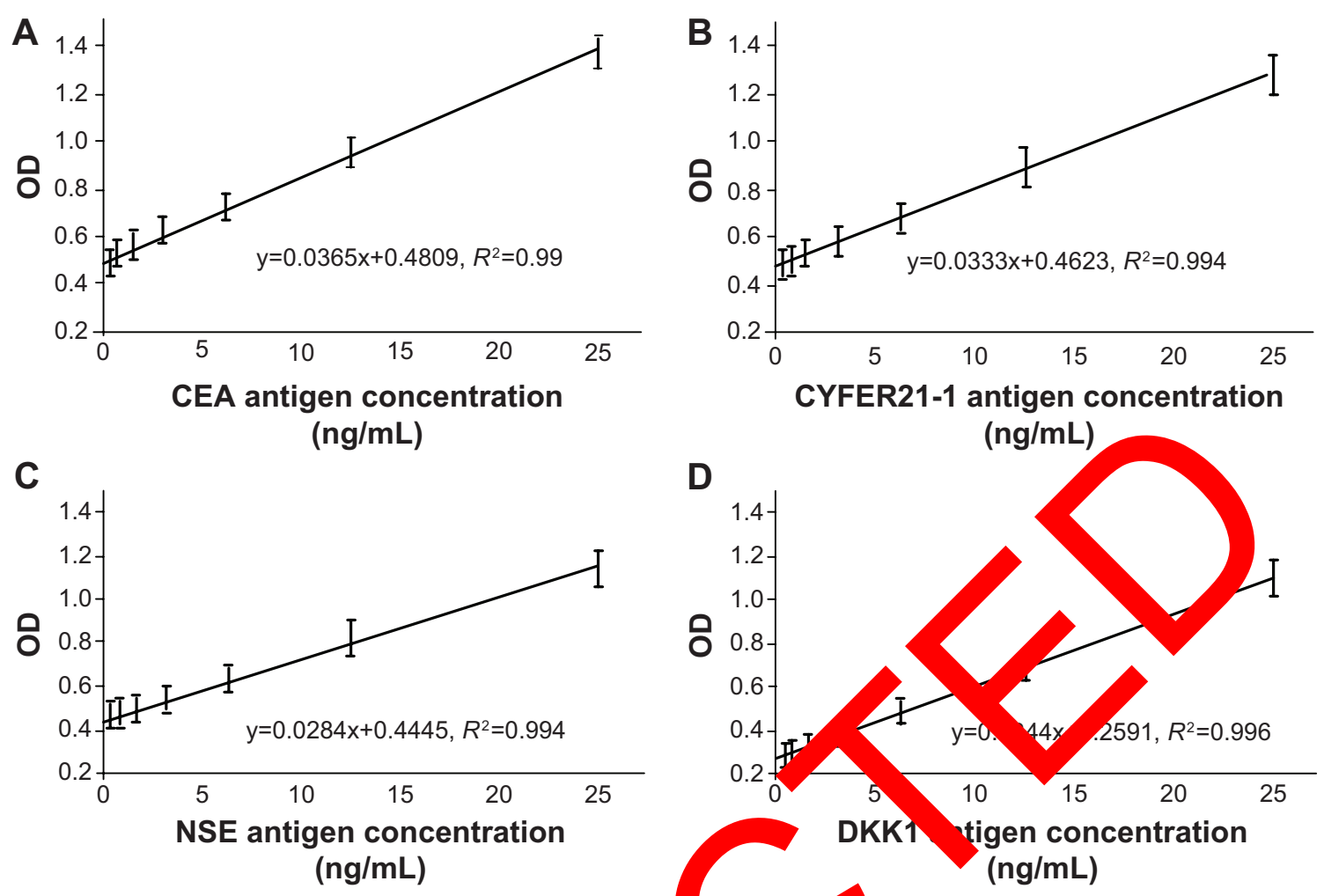

Figure 3 Standard graphs showing the relationship between the four tumor markers a Notes: (A) Antigen concentration of CEA $(\mathrm{ng} / \mathrm{mL}),(B)$ antigen concentration of CYFR2 of DKKI $(\mathrm{ng} / \mathrm{mL})$.

Abbreviations: CEA, carcinoembryonic antigen; CYFRA2I-I, cytokerat

shown in Figure 5A. The relative values we those from ECLIA used in clinical pract gram for CEA is shown in Figure 5B. showed that the detection results these $t$ methods were consistent (relative coefficien or $r=0.986$; YFRA21-1: $r=0.985$; NSE: $r=0.978$; DKK $1: r=0 \quad 93$ respectively).

The four tumor $\mathrm{m}$ ers in the 128 . ym samples were found not to foll a nor 1 distribution (all $P<0.05$ ). Comparing the lun ar group and the healthy control group (Table 1), the difference in the four tumor markers was statistically significant (all $P<0.05$ ), with their concentrations being markedly higher in the lung cancer group than in the control group.

We took $95 \%$ of the values for the four tumor markers from the 42 healthy controls as the critical value $(4.82 \mathrm{ng} / \mathrm{mL}$ for CEA, $3.04 \mathrm{ng} / \mathrm{mL}$ for CYFRA21-1, $23.7 \mathrm{ng} / \mathrm{mL}$ for NSE, and $14.15 \mathrm{ng} / \mathrm{mL}$ for DKKl). We then analyzed the positive detection rate for the four tumor markers in the patients with

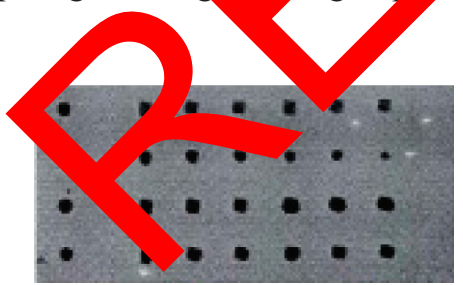

Positive control

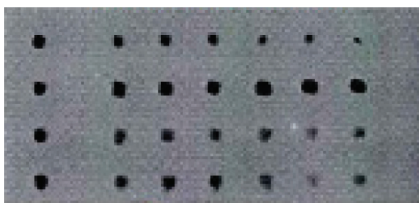

Patient 1

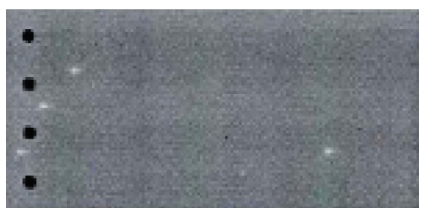

Blank control

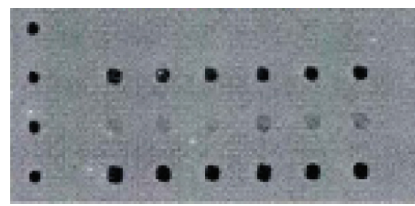

Patient 2

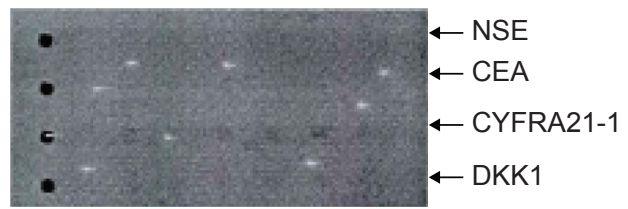

Healthy donors control

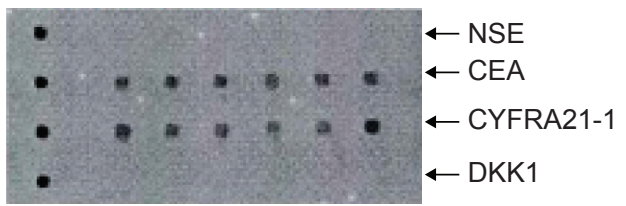

Patient 3

Figure 4 Sample of detection results for the four tumor markers.

Abbreviations: CEA, carcinoembryonic antigen; CYFRA2I-I, cytokeratin-19 fragments; NSE, neuron-specific enolase; DKKI, Dickkopf-I. 
A

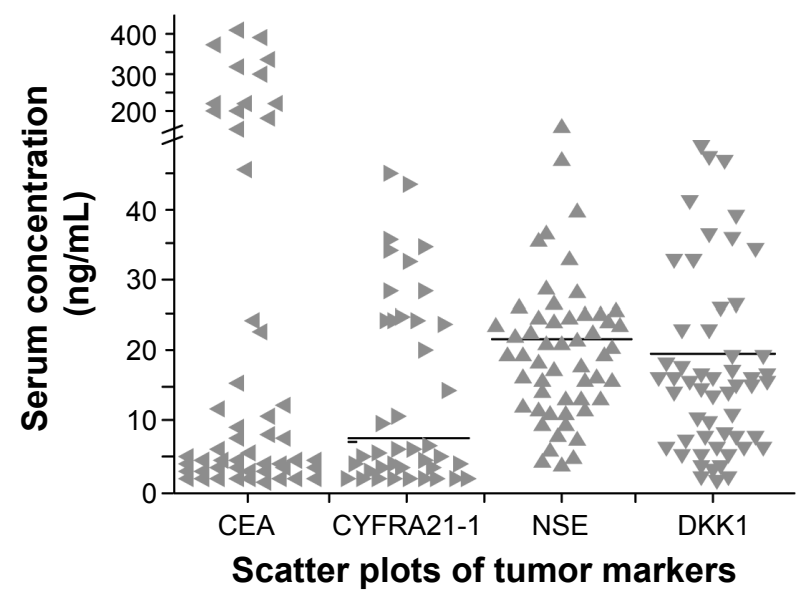

B

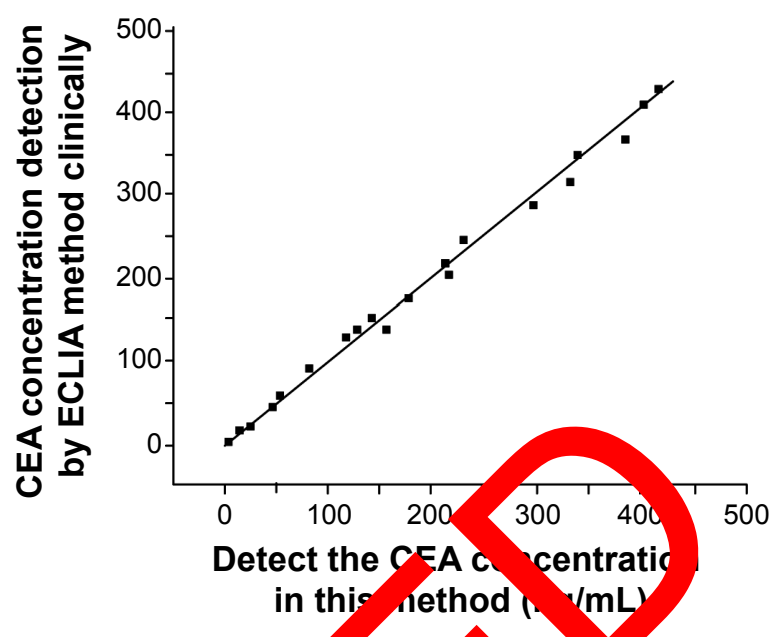

Figure 5 Distribution of the concentrations of four tumor markers $(\mathbf{A})$ and comparison of CEA concentrations detected the metho this study al by electrochemistry method (B). Abbreviations: CEA, carcinoembryonic antigen; CYFRA2I-I, cytokeratin-19 fragments; ECLIA, electrochemil, nescence
DKKI, Dickkopf-I.

lung cancer. Table 2 shows that in the lung cancer group the sensitivity was $38.37 \%$ for CEA, $51.16 \%$ for CYFRA21-1, $26.745 \%$ for NSE, and $52.33 \%$ for DKK1. The joint detection rate for the four tumor markers was $88.37 \%$, which was much higher than any single detection (all $P<0.001$ ). sensitivity of CEA in glandular cancer was $54.35 \%$, and the of CYFRA21-1 in squamous cancer and NSE in cancer was $54.84 \%$ and $66.67 \%$, respectively

\section{Discussion}

CEA is a glycoprotein that is di buted on th colonic mucosal epithelium of the emb 0 and expressed $m$ high levels in several malignay $c$ s, includins ertain gastric, lung, breast, and ovaria cancers Detection changes in serum CEA can be us 1 for Aly diagnosis of malignant tumors, monitorino the ro nse to to ment, and assisting with the prog sis, $\mathrm{D}$ ich is bad for patients showing progre ve incr ses in CE. ${ }^{8}$ CYFRA21-1, a scaffold protein in no, or malignant epithelium, is found in the epithel nolayers an is present in high concentrations in ty rors originating from epithelial tissues. CYFR is also a sen ive tumor $\mathrm{m}$ ker, and has high specificity in squamous canc Serum FRA21-1 levels tends to rise with the increasing or stage, and can predict the prognosis and de the effects of treatment. It has been reported that ont detection of CEA and CYFRA-21 could effectively improve the rate of accurate diagnosis of non-small cell ang cancer. ${ }^{9}$ NSE plays an important role in the diagnosis of small cell lung cancer and a high serum NSE concentration aids in the diagnosis of small cell lung cancer and in the identification and diagnosis of neuroendocrine tumors. There is some evidence that combined measurement of NSE and pro-gastrin-releasing peptide levels can help to identify. ${ }^{10}$ DKK1 is a secretory glycoprotein including a signal peptide sequence and two domains of cysteinamine, which has come to the attention of researchers just recently. It is a secretory protein firstly found by the Dickkopf family. It acts as an inhibitor of wnt $/ \beta$-catenin signals, which

Table I Concentration of four tumor markers in controls and patients with lung cancer

\begin{tabular}{llllll}
\hline & $\mathbf{n}$ & CEA & CYFRA2I-I & NSE & DKKI \\
\hline Healthy donors & 42 & 2.01 & 1.36 & 13.56 & 8.48 \\
& & $(1.29-3.18)$ & $(1.03-2.03)$ & $(10.51-15.86)$ & $(4.91-14.4)$ \\
Patients with lung cancer & 86 & 4.15 & 3.06 & 15.77 & 14.20 \\
& & $(2.78-17.06)$ & $(1.92-5.37)$ & $(11.43-23.76)$ & $(5.75-20.33)$ \\
$Z$ & & -5.633 & -6.11 & -2.416 & -3.311 \\
$P$ & & $<0.001$ & $<0.001$ & 0.016 & 0.001 \\
\hline
\end{tabular}

Notes: Values are expressed as the median [interquartile range] in $\mathrm{ng} / \mathrm{mL}$.

Abbreviations: CEA, carcinoembryonic antigen; CYFRA2I-I, cytokeratin-19 fragments; NSE, neuron-specific enolase; DKKI, Dickkopf-I. 
Table 2 Sensitivity of four tumor markers in patients with lung cancer according to pathological typing

\begin{tabular}{lllllll}
\hline & $\mathbf{n}$ & CEA & CYFRA2I-I & NSE & DKKI & Joint detection \\
\hline Lung cancer & 86 & $38.37(33 / 86)$ & $5 I .16(44 / 86)$ & $26.74(23 / 86)$ & $52.33(45 / 86)$ & $88.37(76 / 86)$ \\
Squamous & 31 & $19.35(6 / 31)$ & $54.84(17 / 31)$ & $25.8 I(8 / 31)$ & $54.84(17 / 31)$ & $87.1(27 / 31)$ \\
Glandular & 46 & $54.35(25 / 46)$ & $52.17(24 / 46)$ & $19.57(9 / 46)$ & $56.52(26 / 46)$ & $91.3(42 / 46)$ \\
Small cell & 9 & $22.22(2 / 9)$ & $33.33(3 / 9)$ & $66.67(6 / 9)$ & $22.22(2 / 9)$ & $77.78(7 / 9)$ \\
\hline
\end{tabular}

Note: Data are shown as sensitivity (the number of samples that have sensitivity of tumor marker/the total amount of samples).

strictly controls the functional status of these signals, and these signals play an important role in the development of stem cells of adults and the regulation system. There have been studies showing markedly decreased serum DKK1 concentrations in patients with gastric cancer, colorectal cancer, ovarian cancer, and cervical adenocarcinoma, and a significant increase in expression in other tumors, such as liver cancer, lung cancer, Wilm's tumor, hepatoblastoma, breast cancer, and multiple myeloma. The increase in DKK1 in patients with lung cancer is closely related to the treatment of tumors. ${ }^{11}$

The microarray of protein chips based on the intensity and parallel processing principle of microelectronics can cure a great number of biological samples with biology significance orderly on solid-phase carrier to specifically arrest the effective ingredients in samples. ${ }^{12}$ It also takes advantage of a charge coupled device or laser sc. system to acquire and analyze images, so that a great leal of information can be handled and made ay It has the features of high flux, high sensit ity, an in abil to perform multivariate analysis. Nanos td part called colloidal gold because they ave a d appearance in aqueous solution. With the vique biolos al features, they can be marked on the surace o. any biomorecules and integrate with them co pletely. Mor ver, they are quite easy to detect bece of thry physical reatures, and play an important role he bedicaldetection.

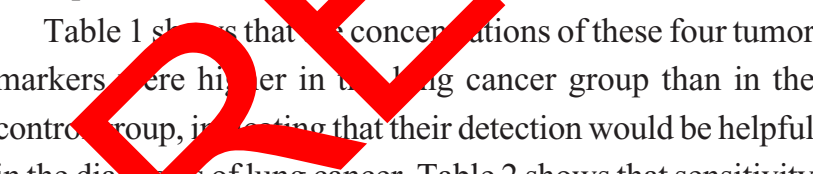
in the dias sof lung cancer. Table 2 shows that sensitivity of CEA for a ction in the lung cancer group was $38.37 \%$, with respective alues of $51.16 \%, 26.74 \%$, and $52.33 \%$ for CYFRA21-1, NSE, and DKK1; the joint detection rate for the four markers was $88.37 \%$, which is higher than that for detection of a single marker $(P<0.0011)$, indicates that joint detection may be useful for diagnosis of lung cancer. The highest sensitivity of CEA in detecting glandular cancer was $54.35 \%$, with values of $54.8 \%$ and $66.67 \%$ for CYFRA $21-1$ in squamous cancer and for NSE in small cell lung cancer, respectively. These findings provide further evidence that CEA is beneficial for detecting glandular cancer, CYFRA21-1 for squamous cancer, and NSE for small cell cancer. DKK1 has no distinct role in the pathological typing of tumors, but it has quite hir wity in all tumor stages as a new tumor marker can gre. y improve the sensitivity in the diagnosis of $\mathrm{r}$ cancer. It important to use joint tumor marke retection sen sc ening for lung cancer and when ating $t^{1}$ diseas With continued developments inscio a lechnology, new techniques and methods for omedica tection ased on nanogold probes are const $A \lambda$ nerging. D this, few can be applied in clinical practice. is is mainly because these techniques will lo comie increase 0 lse positive signals while improving ensitivity dug to to lack of effective control for non-specific gnals. Espe ally during detection of serum samples in c. ic, a gre number of non-specific signal will appear as there and lot of unknown proteins. These problems need a. resolved by further studies. Compared with the single antibody, non-specific absorption particles are easier to clean. This study, taking full advantage of this mechanism, marked the detection antibody on nanogold, which greatly reduced the disturbance signal. Using nanogold as a detection carrier, the detection signal of protein chips and nanogold was magnified. High sensitivity and specificity were ensured, thereby meeting present clinical requirements.

\section{Conclusion}

Using protein chips and nanogold probes, we developed a detection method for ultra-trace protein by magnifying the signal of nanogold deposition. This method enabled visual testing for proteins in a semi-quantitative way, and could detect four tumor markers, ie, CEA, CYFRA21-1, NSE, and DKK1 within 1.5 hours with high sensitivity. It enables simultaneous and rapid detection of multi-tumor markers, enabling early diagnosis and assessment for lung cancer. In this work, we also performed a correlation analysis between the results in this study and the detection results from ECLIA combined with DDK1 kit in clinical practice, and found consistency. The method described here is considered to have a wide application, given that the experimental results can be analyzed simply with the naked eye or an ordinary 
microscope. Comparison of detection results using different devices provides a sound basis for the detection of tumor markers in clinical practice.

\section{Disclosure}

The authors report no conflicts of interest in this work.

\section{References}

1. Siegel R, DeSantis C, Virgo K, et al. Cancer treatment and survivorship statistics. CA Cancer J Clin. 2012;62(4):220-241.

2. Croswell JM, Baker SG, Marcus PM, Clapp JD, Kramer BS. Cumulative incidence of false-positive test results in lung cancer screening: a randomized trial. Ann Intern Med. 2010;152(8):505-512.

3. Hui L, Yanle C, Jingxia C, et al. Value of detection of serum tumor biomarkers in lung cancer diagnosis, pathological type and clinical stage. Chinese Journal of Lung Disease (Electronic Edition). 2013;6(2): 144-148.

4. Wen G, Yongqian S. New progress of medical treatment of advanced non-small cell lung cancer. Chinese Journal of Clinicians (Electronic Edition). 2013;7(18):11-16.

5. Huimin W, Hua Z, Bo J, et al. Clinical value of serum tumor markers in predicting patients' responses to targeted therapy for advanced non-small cell lung cancer. Tumor. 2012;32(012):1021-1024.
6. Bigbee WL, Gopalakrishnan V, Weissfeld JL, et al. A multiplexed serum biomarker immunoassay panel discriminates clinical lung cancer patients from high-risk individuals found to be cancer-free by CT screening. J Thorac Oncol. 2012;7(4):698-708.

7. Tianlei $\mathrm{N}$. The technology and its feature of chemiluminescent immunoassay (CLIA). Modern Medicine Health. 2011;27(14):2156-2158.

8. Lina Z, Jianli M, Dianjin H, et al. Clinical significance of CYFRA21-1 and CEA in the early diagnosis of patients with locally advanced nonsmall cell lung cancer. Modern Oncology. 2013;21(3):549-551.

9. Changbing J, Bangping C, Wenli D, et al. The value of joint detection of CEA, NSE, CYFRA21-1 in diagnosis and treatment of lung cancer. Chongqing Medical Journal. 2013;42(3):283-284.

10. Xiaxia J, Shiyong C, Juanfei Q, et al. Diagnostic significance of joint detection on serum ProGRP, NSE, CEA, SCCA and CYFRA21-1 in patients with lung cancer. Journal of Radi 283-284.

11. Xiaohong H, Jinhui Y. Progress of K-1 and tumb Journal of Chengdu Medical College. 2014:9(3):3 369.

12. Lili J, Chenpeng Z, Xiaoguay 2 , et al. lication o nulti-tumor marker protein chip in he? examination. H, un sical Journal. 2014;30(4):342-346.

13. Bo J, Qifei W, YushrM, A cation value of joint detection of tumor markers in sted dia sis of lung acer. Journal of Clinical Medicine in $\mathrm{Py}$

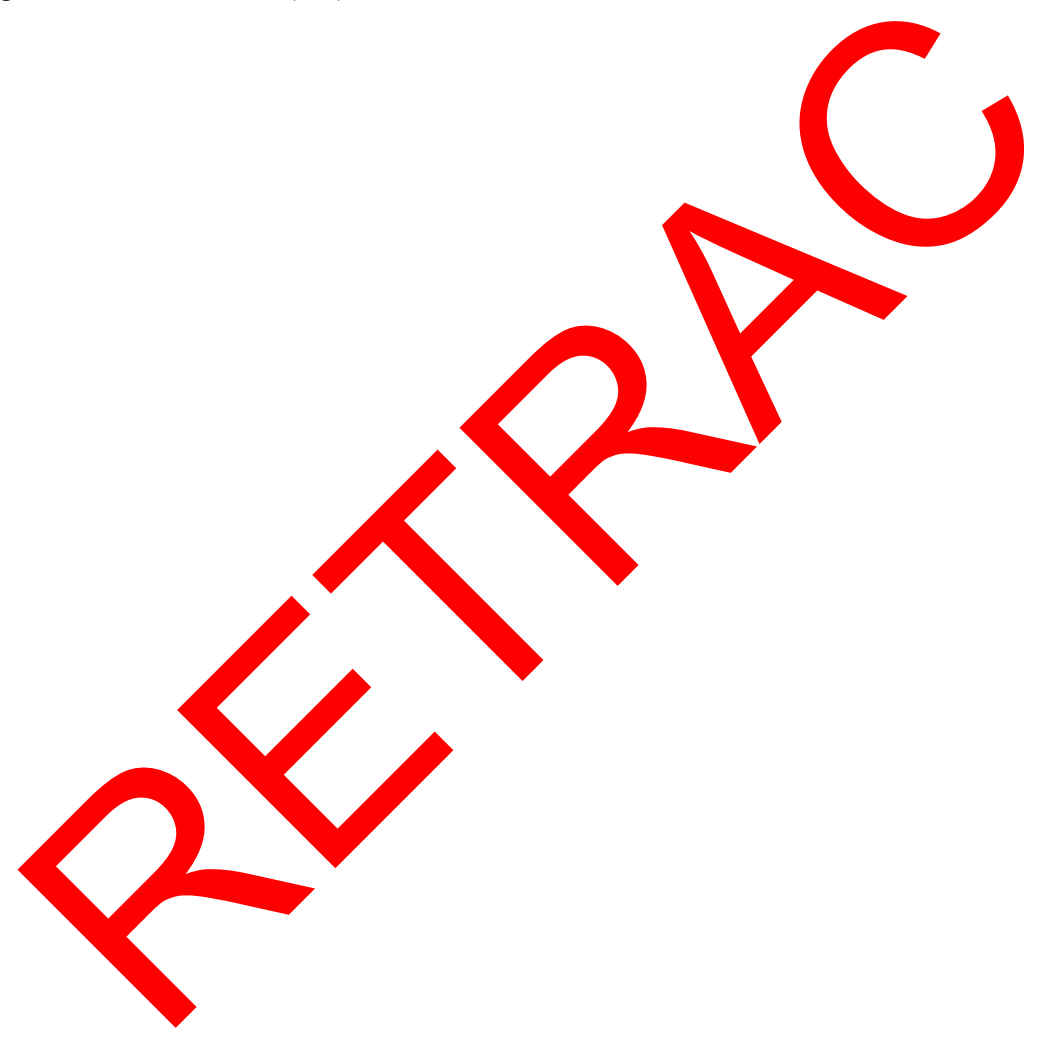

OncoTargets and Therapy

Dovepress

\section{Publish your work in this journal}

OncoTargets and Therapy is an international, peer-reviewed, open access journal focusing on the pathological basis of all cancers, potential targets for therapy and treatment protocols employed to improve the management of cancer patients. The journal also focuses on the impact of management programs and new therapeutic agents and protocols on patient perspectives such as quality of life, adherence and satisfaction. The manuscript management system is completely online and includes a very quick and fair peer-review system, which is all easy to use. Visit http://www.dovepress.com/testimonials.php to read real quotes from published authors. 